\title{
Primary Alveolar Soft Part Sarcoma of the Scapula
}

\author{
Ghada Sahraoui, Nadia Boujelbene, Raoudha Doghri, Karima Mrad \\ Department of Pathology, Salah Azaiez Institute, Tunis, Tunisia \\ Email: ghada_sahraoui@yahoo.com
}

How to cite this paper: Sahraoui, G., Boujelbene, N., Doghri, R. and Mrad, K. (2017) Primary Alveolar Soft Part Sarcoma of the Scapula. Open Journal of Orthopedics, 7 , 7-13.

http://dx.doi.org/10.4236/ojo.2017.71002

Received: December 8, 2016

Accepted: January 19, 2017

Published: January 22, 2017

Copyright $\odot 2017$ by authors and Scientific Research Publishing Inc.

This work is licensed under the Creative

Commons Attribution International

License (CC BY 4.0).

http://creativecommons.org/licenses/by/4.0/

\begin{abstract}
Alveolar soft-part sarcoma (ASPS) is an uncommon, aggressive soft-tissue neoplasm, occurring in less than $1 \%$ of sarcomas. It is typically found in the head and neck tissues in children or in the deep soft tissues of the lower extremities in young adults. This tumor arises primarily within the skeletal muscles or musculofascial planes. Primary involvement of bone is extremely rare. ASPS has poor prognosis with a propensity for recurrence as well as distant metastasis. In this report, we present a 25-year-old male with primary ASPS in the left scapular bone and discuss the histopathological and immunohistochemical features of this sarcoma.
\end{abstract}

\section{Keywords}

Alveolar Soft-Part Sarcoma, Histopathological, Scapula

\section{Introduction}

Alveolar soft part sarcoma (ASPS) is rare, distinctive soft tissue sarcoma composed of large, uniform, epithelioid cells and characterized by ASPSCR1-TFE3 fusion gene [1]. It accounts for less than $1 \%$ of all soft tissue malignancies, and generally occurs between 15 and 35 years of age with a notable female predominance [1] [2]. ASPS usually presents as a slowly growing, painless mass [1]. Pediatric ASPS appears most commonly in the head and neck tissues, however, lower extremity involvement may be found in young adults [3]. Primary bone involvement is occasional, and characteristically involves the fibula, ileum and tibia [4]. Only 2 cases with primary involvement of the scapular bone have been reported previously in the literature [3]. This case highlights some of the histopathological pitfalls in the diagnosis of ASPS and the possible unusual site of presentation. 


\section{Case Report}

A 25-year-old male presented with a rapidly increasing swelling over the left scapular region of six months duration. Physical examination detected a $9.5 \times 9$ $\mathrm{cm}$ sized scapular tumor fixed to the deep structures, but free from the overlying skin and a limited mobility of the left shoulder. Contrast-enhanced computed tomography (CT) of the left shoulder revealed a heterogeneous mass within the left scapula resulting in destruction of the bone cortex and local expansion to adjacent muscle, bone and joint structures. Magnetic resonance imaging (MRI) showed an osseous soft tissue mass with necrotic center originating in the left scapular region and involving the rotator cuff muscles and the gleno-humeral joint (Figure 1). Thoracoabdominal CT showed metastatic lung, spleen and lymph node lesions.

Tru-Cut biopsy of the scapular lesion was performed. Microscopic exam showed cellular tumor arranged in a nesting or pseudoalveolar pattern of largerelatively uniformepithelioid cells with abundant eosinophilic granular cytoplasm and hyperchromatic vesicular nuclei having prominent nucleoli (Figure 2, Figure 3). There was a mitotic rate of 1 per ten high power fields. According to these morphological features, both rhabdomyosarcoma and alveolar soft part sarcoma were suspected. Immunohistochemically, the tumor cells showed positive staining for Desmin (Figure 4) and a strong nuclear staining for TFE3 (Figure 5). Staining for CD31, epithelial markers and myogenin were negative.

Morphological features and immunohistochemical stains were in favor of the diagnosis of ASPS.

Since the tumor was judged non-resectable by surgeons, the patient received palliative chemotherapy (Doxorubicin $106 \mathrm{mg} /$ day; Ifosfamide $5 \mathrm{~g}$ the first day and 5, $6 \mathrm{~g}$ the second day; Mesna $6 \mathrm{~g}$ the first day and $7 \mathrm{~g}$ the second day) but

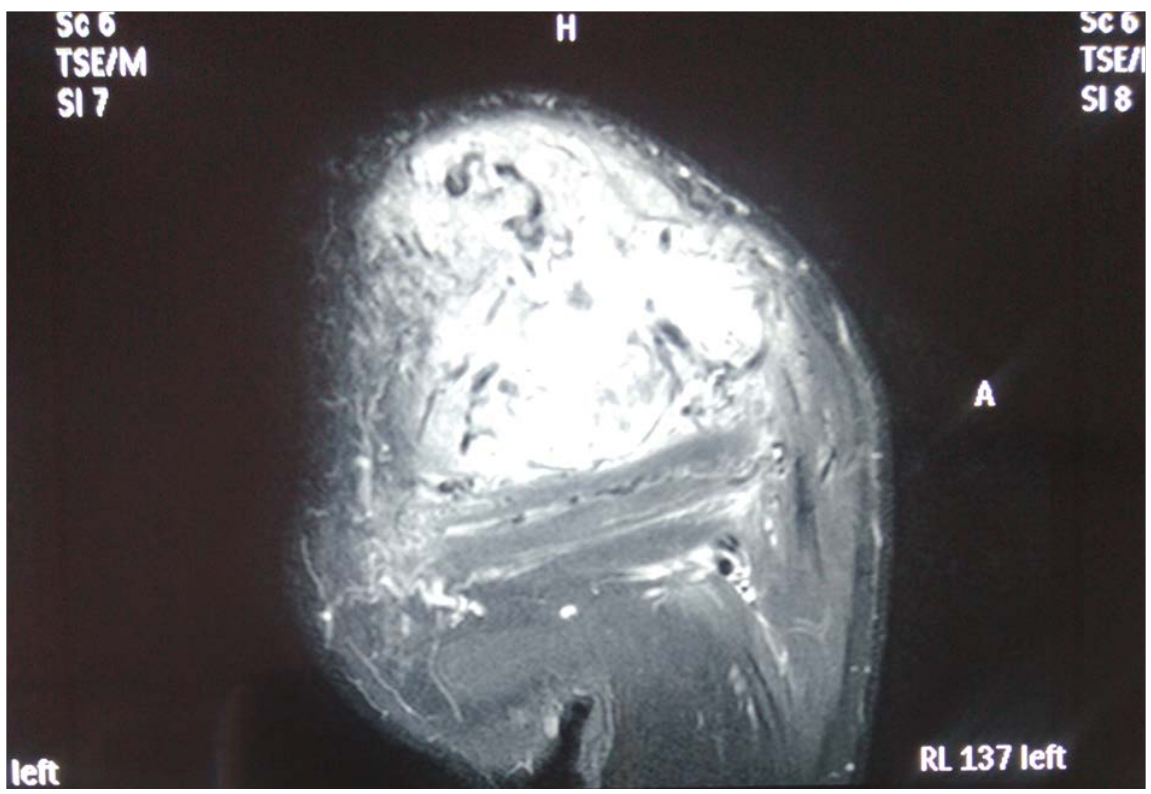

Figure 1. MRI of the left shoulder: hyperintense osseous soft tissue mass involving the rotator cuff muscles and the gleno-humeral joint. 


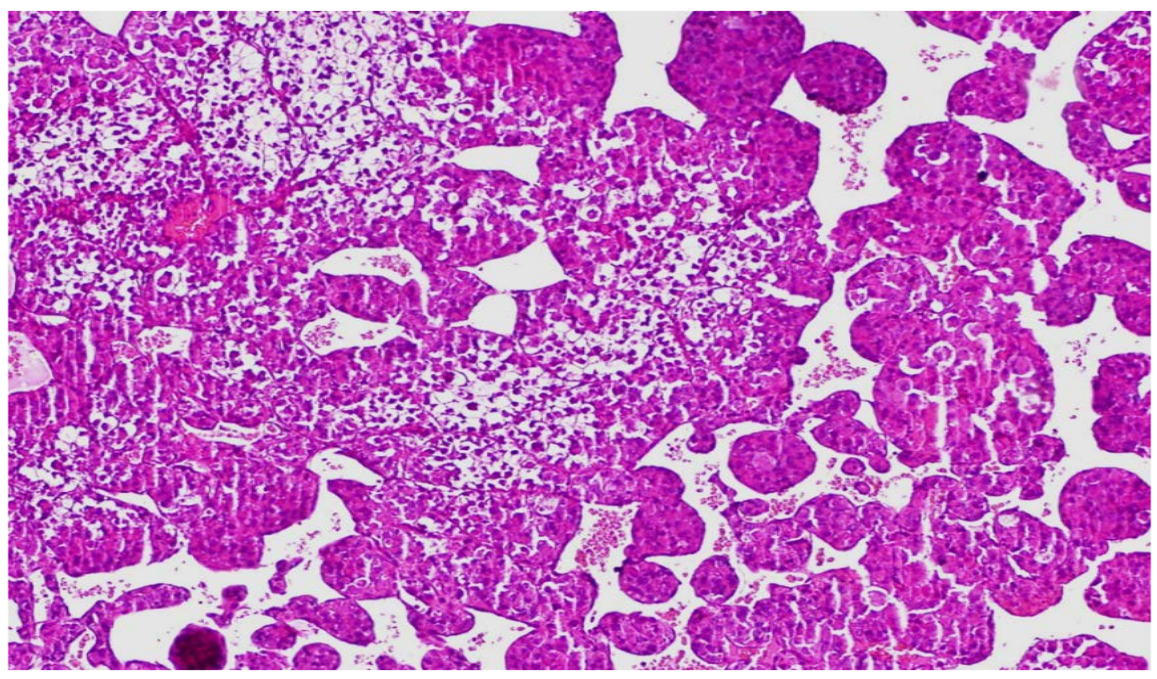

Figure 2. Alveolar nests of tumor cells separated by thin fibrous septae.

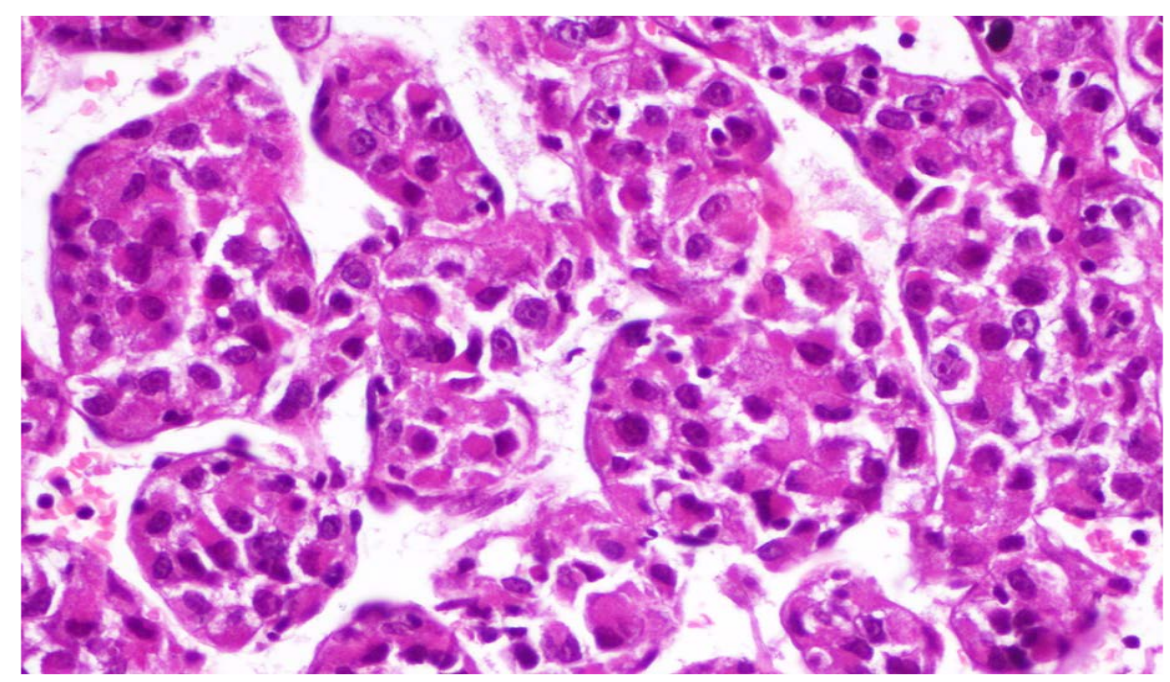

Figure 3. Epithelioid cells with abundant eosinophilic granular cytoplasm.

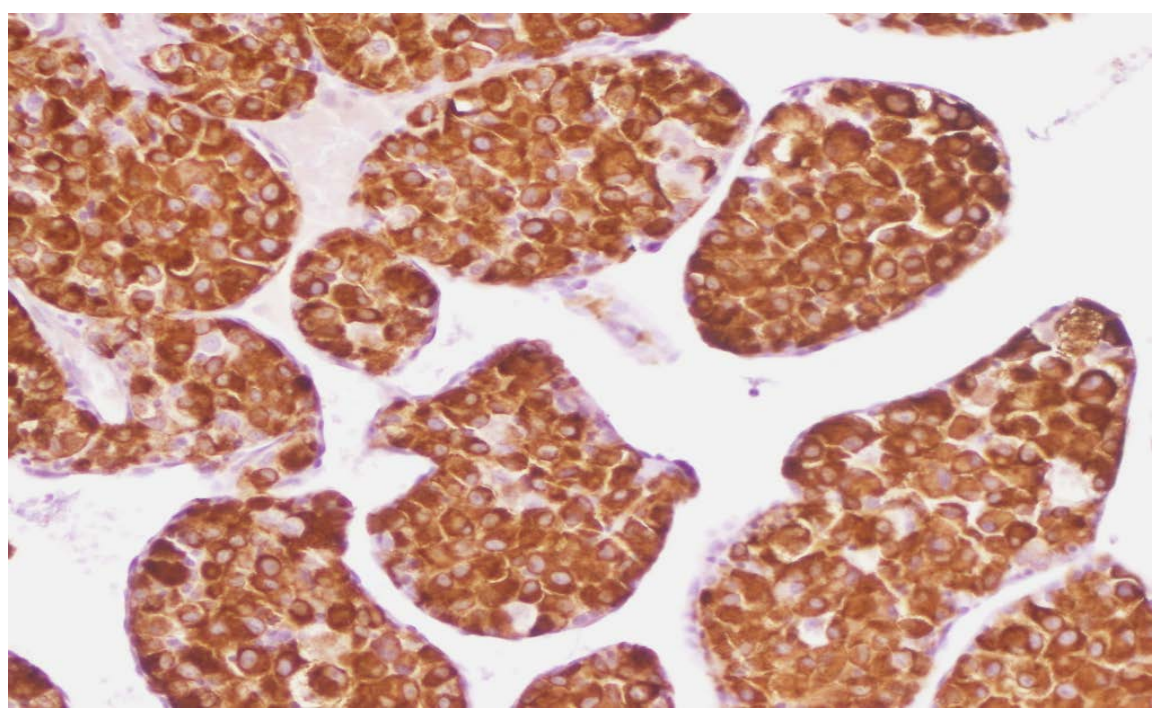

Figure 4. Tumor cells express desmin. 


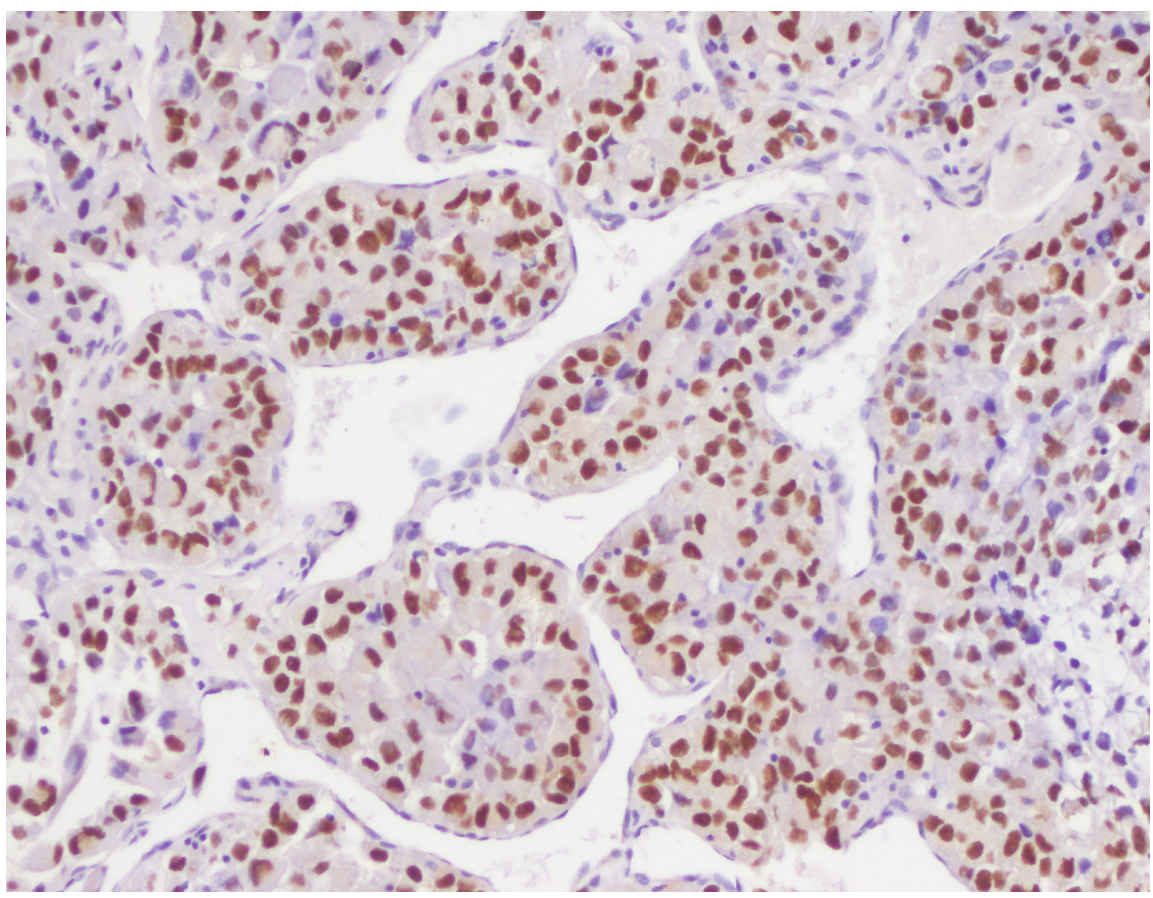

Figure 5. Strong nuclear staining for TFE3.

without any response. The metastatic lesions detected at the beginning of the explorations persisted and other cerebral and hepatic metastases had appeared. Another regimen of chemotherapy was proposed. However, the patient refused to complete the treatment and died seven months after the latest course of chemotherapy.

\section{Discussion}

ASPS is a rare soft-tissue malignancy that was originally alluded to by Smetana and Scott as a malignant tumor of the non-chromaffin paraganglia in 1951 [5], and then described under its present name of "alveolar soft-part sarcoma" by Christopherson et al. in 1952 [1]. It occurs, in less than $1 \%$ of sarcomas, usually in adolescents and young adults, with a female predominance [3] [6]. ASPS primarily arises from the deep soft tissue of the extremities, trunk, retroperitoneum, head and neck. In children, ASPS has an unusual tendency to involve the tongue and orbit [7] [8]. Other locations, such as the female genital tract [6], mediastinum [9], lung [10], gastrointestinal tract, are rare [11]. Primary bone involvement is occasional [12], and it characteristically involves the fibula, ileum and tibia [4]. Only 2 cases with primary involvement of the scapular bone have been previously reported in the literature [3]. Radiologic findings reported in these cases were similar to ours: ill-defined osteolytic lesions on plain radiographs and expansile bone tumor with large intra- and extraosseous soft tissue masses on CT [3]. Park and al. [13] described a common feature of all cases which is bone destruction with poorly defined tumor margins. The pathological diagnosis of ASPS is based on morphological features, including large, uniform, epithelioid cells with eosinophilic abundant granular cytoplasm arranged in sol- 
id nests and/or alveolar structures [14]. The distinctively organoid or nesting may be absent especially in children. The nests tend to be uniform but may vary in size and shape. Loss of cellular cohesion and necrosis of centrally located cells in the nests result in the commonly seen pseudoalveolar pattern. The neoplastic cells are generally uniform in size, round or polygonal, with well-defined borders conferring an epithelioid appearance. The nucleus is generally centrally placed, bland with prominent nucleolus. Multinucleation and nuclear atypia are uncommon. Mitotic figures are infrequent. The cytoplasm is abundant eosinophilic and granular but occasionally may exhibits some clear features that mimics renal clear cell carcinoma. The cytoplasm may also contain rhomboid or rod-shaped inclusions that can be faintly apparent in standard coloration but better demonstrated on periodic acid Schiff (PAS) stains after diastase digestion. In addition to that, vascular invasion is frequently seen. Morphologically, our tumor met all these criteria but PAS stains were negative.

Because of the young age of our patient, the clinical presentation and the histological aspect of the tumor, rhabdomyosarcoma was the first diagnostic evoked which justified the first panel of immunohistochemistry based on the antibodies Myogenin and Desmin. The negativity of myogenin prompted us to seek another diagnosis. Thanks to the positivity of the anti-TFE3 carried out in the second panel of immunohistochemistry, the exact diagnosis was made.

The most characteristic finding in ASPS is the consistent demonstration of strong nuclear immunoreactivity for TFE3, reflecting the ASPSCR1-TFE3 fusion gene that had been identified by cytogenetic analysis [4] [14]. Recent studies have shown that the crystals are immunoreactive to a protein MCT1 (monocarboxylate transporter) and to CD 147 [15]. About 50\% of ASPS tumors express Desmin, as our case showed. Staining for protein S100 can be positive. Generally, epithelial, melanocytic, neural, glial, and neuroendocrine markers are negative [14] [16].

The differential diagnosis of ASPS [4] [17] involves a wide range of neoplasms that contain a similar alveolar nest-like pattern. This includes renal, adrenal and hepatocellular carcinomas, which have a similar eosinophilic cytoplasm, but are distinguished by cytokeratin markers.

Melanoma can be differentiated by PAS negativity and positivity for melanocyticmarkers. Unlike ASPS, extra adrenal paragangliomas show strong expression of neuroendocrine markers and S100 typically is positive in the sustentacular cells. Granular cell tumors are strongly positive for S100, and lack the characteristic vascularization of ASPS.

Due to the alveolar architecture and occasional staining for muscle markers, a diagnosis of alveolar rhabdomyosarcoma may be considered, however, the cytology of ASPS is larger and with more abundant cytoplasm than seen in alveolar rhabdomyosarcomas. While both are highly vascularized tumors, the differentiation of ASPS from hemangioendothelioma is not commonly a diagnostic dilemma. Renal cell carcinoma occasionally mimics ASPS, but often shows focal tubular pattern of growth and lacks the crystals. 
It is reported that ASPS is an indolent disease with characteristic slow growth, but that is associated with a poor overall outcome and a 5-year survival rate of only $20 \%$ in unresectable metastatic patients [18]. Unlike the rare cases described in the literature where patients have undergone surgical treatment and been relatively improved after treatment [3], our patient had rapidly fatal outcome.

Metastasis can occur early sometimes before primary lesion detection or later after tumor resection even without local recurrence [14]. The most common sites of metastases are, in decreasing order of frequency, lung, bone and brain [14]. Metastases to the lymph nodes are not frequent [14]. Prognostic factors are age at presentation, tumor size and the presence of metastasisat diagnosis [14]. Tumor size correlates with metastatic disease at onset and is the major factor influencing survival [19].

Surgical excision remains the mainstay of therapy, and adjuvant chemotherapy and radiotherapy have no definite role in its management [15] [19]. In the present case the tumor was found to be infiltrating adjacent muscles therefore judged inoperable. The patient received adjuvant chemotherapy and continues to be on follow up.

\section{Conclusion}

In conclusion, this case is presented to highlight the distinctive clinical, morphological and immunohistochemical features of this unusual tumor. ASPS should be taken into consideration in the differential diagnosis of bone-originating soft tissue masses. Characteristic features of the lesion contribute to differentiating this entity from other bone tumors, primitive or metastatic.

\section{References}

[1] Christopherson, W.M., Foote, F.W. and Stewart, F.W. (1952) Alveolar Soft-Part Sarcomas; Structurally Characteristic Tumors of Uncertain Histogenesis. Cancer, 5 , 100-111.

https://doi.org/10.1002/1097-0142(195201)5:1<100::AID-CNCR2820050112>3.0.C $\underline{\mathrm{O} ; 2-\mathrm{K}}$

[2] Chen, Z., Chengtao, S., Wei, S., et al. (2016) Alveolar Soft-Part Sarcoma in the Left Forearm with Cardiac Metastasis: A Case Report and Literature Review. Oncology Letters, 11, 81-84.

[3] Yavuz, A., Cemil, G., Aydin, B. and Mehmet, B. (2013) Primary Alveolar Soft Part Sarcoma of the Scapula. Case Reports in Oncology, 6, 356-361. https://doi.org/10.1159/000353927

[4] James, A.W., Chang, L., Levine, B. and Dry, S.M. (2014) Clavicular and Meningeal Alveolar Soft Part Sarcoma: An Unusual Case and Literature Review. Journal of Orthopaedics, 11, 48-53. https://doi.org/10.1016/j.jor.2013.12.014

[5] Smetana, H.F. and Scott, W.F. (1951) Malignant Tumors of Nonchromaffin Paraganglia. Military Surgeon, 109, 330-349.

[6] Zhang, L., Tang, Q., Wang, Z. and Zhang, X. (2012) Alveolar Soft Part Sarcoma of the Uterine Corpus with Pelvic Lymph Node Metastasis: Case Report and Literature Review. International Journal of Clinical and Experimental Pathology, 5, 715-719. 
[7] Fanburg-Smith, J.C., Miettinen, M., Folpe, A.L., Weiss, S.W. and Childers, E.L.B. (2004) Lingual Alveolar Soft Part Sarcoma; 14 Cases: Novel Clinical and Morphological Observations. Histopathology, 45, 526-537. https://doi.org/10.1111/j.1365-2559.2004.01966.x

[8] Font, R.L., Jurco, S. and Zimmerman, L.E. (1982) Alveolar Soft-Part Sarcoma of the Orbit: A Clinicopathologic Analysis of Seventeen Cases and a Review of the Literature. Human Pathology, 13, 569-579. https://doi.org/10.1016/S0046-8177(82)80273-6

[9] Flieder, D.B., Moran, C.A. and Suster, S. (1997) Primary Alveolar soft-Part Sarcoma of the Mediastinum: A Clinicopathological and Immunohistochemical Study of Two Cases. Histopathology, 31, 46-73. https://doi.org/10.1046/j.1365-2559.1997.2690871.x

[10] Trabelsi, A., Ben Abdelkrim, S., Taher, Y.M., Mlika, S., Hmissa, S., Mokni, M., et al. (2009) Primary Alveolar Soft Part Sarcoma of the Lung. Revue des Maladies Respiratoires, 26, 329-332. https://doi.org/10.1016/S0761-8425(09)72591-4

[11] Yaziji, H., Ranaldi, R., Verdolini, R., Morroni, M., Haggitt, R. and Bearzi, I. (2000) Primary Alveolar Soft Part Sarcoma of the Stomach: A Case Report and Review. Pathology-Research and Practic, 196, 519-525. https://doi.org/10.1016/S0344-0338(00)80054-0

[12] Das, K.K., Singh, R.K., Jaiswal, S., Agrawal, V., Jaiswal, A.K. and Behari, S. (2012) Alveolar Soft Part Sarcoma of the Frontal Calvarium and Adjacent Frontal Lobe. Journal of Pediatric Neurosciences, 7, 36-39. https://doi.org/10.4103/1817-1745.97621

[13] Park, Y.K., Unni, K.K., Kim, Y.W., Han, C.S., Yang, M.H., Wenger, D.E., et al. (1999) Primary Alveolar Soft Part Sarcoma of Bone. Histopathology, 35, 411-417. https://doi.org/10.1046/j.1365-2559.1999.035005411.x

[14] Ordónĕz, N.G. (2013) Alveolar Soft Part Sarcoma. In: Fletcher, C.D.M., World Health Organization Classification of Tumours of Soft Tissues and Bone, IARC Press, Lyon, 218-221.

[15] Weiss, S.W. (2002) Alveolar Soft Part Sarcoma: Are We at the End or Just the Beginning of Our Quest? The American Journal of Pathology, 160, 1197-1199. https://doi.org/10.1016/S0002-9440(10)62545-X

[16] Aluigi, P., Sangiorgi, L. and Picci, P. (1996) Alveolar Soft Part Sarcoma. Skeletal Radiology, 25, 400-402. https://doi.org/10.1007/s002560050104

[17] Nair, A., Pai, D.R., Jagdish, S. and Krishnan, R. (2003) Alveolar Soft Part Sarcoma: A Unique Tumor with Disputed Histogenesis. Indian Journal of Cancer, 40, 82.

[18] Shivaani, K, Deborah, A. and Helman, L. (2013) Cediranib for Metastatic Alveolar Soft Part Sarcoma. Journal of Clinical Oncology, 31, 2296-2302.

http://www.ncbi.nlm.nih.gov/pmc/articles/PMC3677840/ https://doi.org/10.1200/JCO.2012.47.4288

[19] Casanova, M., Ferrari, A., Bisogno, G., Cecchetto, G., Basso, E., De Bernardi, B., et al. (2000) Alveolar Soft Part Sarcoma in Children and Adolescents: A Report from the Soft-Tissue Sarcoma Italian Cooperative Group. Annals of Oncology, 11, 14451449. https://doi.org/10.1023/A:1026579623136 
Submit or recommend next manuscript to SCIRP and we will provide best service for you:

Accepting pre-submission inquiries through Email, Facebook, LinkedIn, Twitter, etc. A wide selection of journals (inclusive of 9 subjects, more than 200 journals)

Providing 24-hour high-quality service

User-friendly online submission system

Fair and swift peer-review system

Efficient typesetting and proofreading procedure

Display of the result of downloads and visits, as well as the number of cited articles Maximum dissemination of your research work

Submit your manuscript at: http://papersubmission.scirp.org/

Or contact ojo@scirp.org 